\title{
Field Trial of New Technologies for Reducing Mosquito Populations in Bulloch County, Georgia, USA
}

\author{
Thomas M Kollars ${ }^{1 *}$ and Bannie Hulsey ${ }^{2}$ \\ ${ }^{1}$ Health Sciences, Lynchburg, VA, USA \\ ${ }^{2}$ Crisp Academy, USA \\ *Corresponding author: Thomas M Kollars, Health Sciences, VA, USA
}

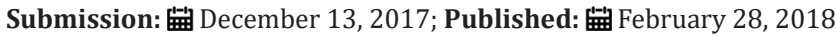

\begin{abstract}
Mosquito-borne diseases such as West Nile virus and Eastern Equine Encephalitis virus pose an important health risk to residents and travelers to southeastern Georgia. There is an increasing threat of introduction of mosquito-borne diseases, such as Zika and dengue virus, into Georgia where susceptible mosquito species are present. A field trial of the ProVector Tube Applicator with Entobac D pesticide was conducted to determine if the system was effective in reducing mosquito populations in Bulloch County, located in southeastern Georgia. Within one month, there was a significant difference in reduction of mosquitoes in the test versus the control sites, $93 \%$ versus $1 \%$, respectively. There was a significant reduction in the test but not the control every two weeks. Providing effective, inexpensive, safe and eco-friendly tools for officials and homeowners is necessary in the event local mosquito control is poorly funded.
\end{abstract}

Keywords: Public health; Zika; Pesticide; Vector control; Malaria

\section{Introduction}

Nearly 4 billion people are at risk from vector-borne diseases (VBD), with VBD's accounting for more than $17 \%$ of all infectious disease cases being caused globally [1]. Malaria, filariasis and arboviruses, such as dengue, yellow fever virus, Chikungunya, Zika, and Ross River infect millions annually. Mosquito-borne diseases also have an impact on domestic animals as well, such as equine encephalitis viruses and heart worm. Mosquito-borne diseases have significant impact on health in the United States, West Nile, Eastern Equine Encephalitis, La Crosse, St. Louis, and dengue occurring in several states. Zika virus is also an important emerging infectious disease in the United States, particularly in Florida and Texas.

Along with habitat and host species, temperature, humidity, precipitation, are all important factors in determining the presence and abundance of mosquito species in Georgia [2]. A number of MBD's occur in Georgia, West Nile virus being the most common, but when cases of Eastern equine encephalitis (EEE) virus occur, the impact on the patient and their family members can be devastating. West Nile virus and EEE are currently a significant health risk in Bulloch County, Georgia. Preventing the introduction of mosquitoborne arboviruses, such as dengue, Chikungunya and Zika, along with the presence of competent vectors poses a challenge to public health officials and families. Many counties in the state of Georgia do not have a robust mosquito control division, Bulloch County is among them. Without an official integrated mosquito management plan, finding cheap, safe, and environmentally friendly options that local officials and citizens can use to rapidly reduce mosquito populations is essential. Here is discussed a new vector control system, consisting of a new applicator and new pesticide, that rapidly reduces mosquito populations and that is flexible enough to be used across habitats and under harsh environmental conditions, and is target specific in delivering a new pesticide to vectors, reducing cost and environmental impact.

\section{Methods}

An initial mosquito collection was conducted to get a sample of mosquito populations before deployment of the ProVector $₫$ Tube (PVT), Figure 1. Mosquitoes were collected at two test and one control site using one CDC trap and two ABC light traps placed at each site during August and September. Each site measured $1 / 2$ hectare. Octenol tabs and $\mathrm{CO}_{2}$ bottle were used as bait on the devices. One CDC and two ABC light traps were also placed, in a similar fashion, at the Control site for two nights each two weeks for one month, Weeks 0, 2 and 4. After the initial mosquito survey, 15 PVT's with Entobac ${ }^{\mathrm{TM}} \mathrm{D}$ (composed of sugar bait, active ingredients Bacillus thuringiensis israelensis (Bti) plus deltamethrin) were placed within a one acre area at two test sites and one control site. Data analysis was conducted using Statistica software. 


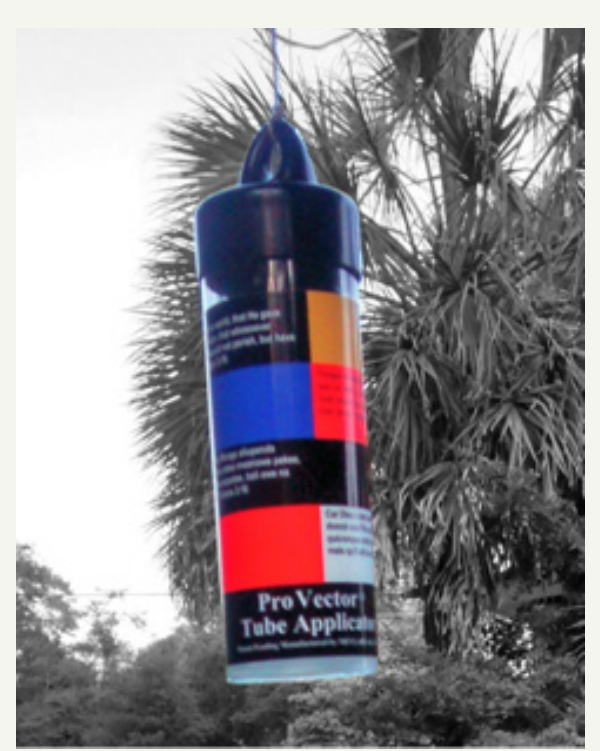

Figure 1: The ProVector ${ }^{\circledR}$ Tube Applicator uses different colors to attract different mosquito species, which feed through holes in the bottom of the tube on an attractant bait, protecting non-target species such as bees and butterflies and reducing pesticide in the environment.

\section{Results and Discussion}

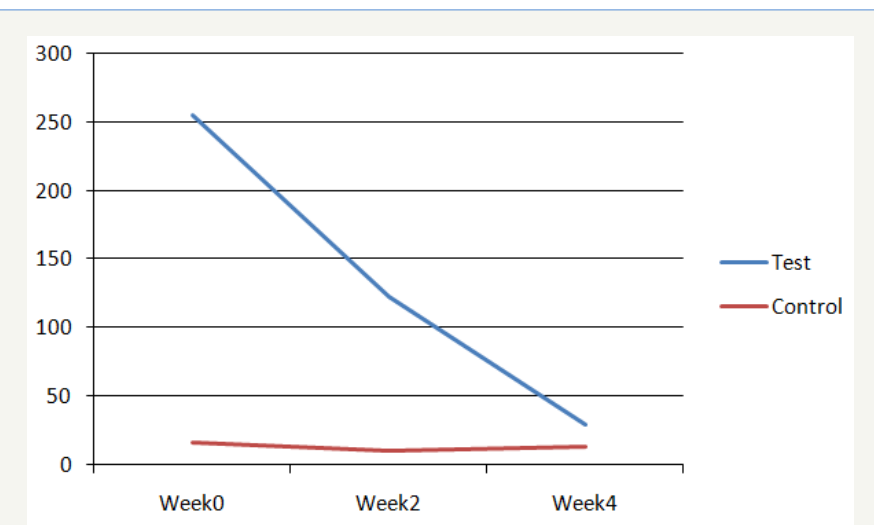

Figure 2: Within one month the ProVector Tubes with Entobac D provided a $93.4 \%$ reduction of mosquitoes where as the control site remained nearly the same with a 0.8 reduction.

Table 1: Chi square analysis of observed verses expected numbers of mosquitoes captured at field sites.

\begin{tabular}{|c|c|c|}
\hline Sites & Chi square Week 0 vs 2 & Chi2 Week 2 vs 4 \\
\hline Test & $1135.94, \mathrm{df}=11, \mathrm{p}<0.05$ & $1410.917, \mathrm{df}=11, \mathrm{p}<0.05$ \\
\hline Control & $1.67, \mathrm{df}=5 \mathrm{p}=.893072$ & $8.500000 \mathrm{df}=5, \mathrm{p}=.13$ \\
\hline
\end{tabular}

There was a significant reduction of mosquitoes at the test sites with the PVT's with Entobac D (Figure 2). At the beginning of the study the average number of mosquitoes captured per trap was 8 times higher at test vs control sites. After 4 weeks the mean number of mosquitoes at the sites was nearly identical, 4.8 vs 4.3 (Figure 2). There was a significant reduction of mosquitoes at the Test but not at the control sites (Table 1). Reducing mosquito populations indoors and outdoors can have a significant impact on malaria and other mosquito-borne diseases. In a study conducted by the Walter Reed Army Institute of Research in Kenya, the ProVector Flower with Entobac (Bti only) was tested against adult mosquitoes. Seven housing compounds with no applicators were included as controls in the study area. After one month, there was a significant reduction of medically important mosquitoes in all nine compounds with the ProVector Flower compared to two of seven control compounds (Chi-square=11.5, p < 0.05) [3]. The ProVector Flower has an open face and must be used either indoors or hung in a way to protect it from rain. The ProVector Tube was developed to be used either indoors or outdoors. The colors on these devices are important as they attract different types of species and where a device is used to control medically important mosquitoes, one should have flexibility (Figure 3).

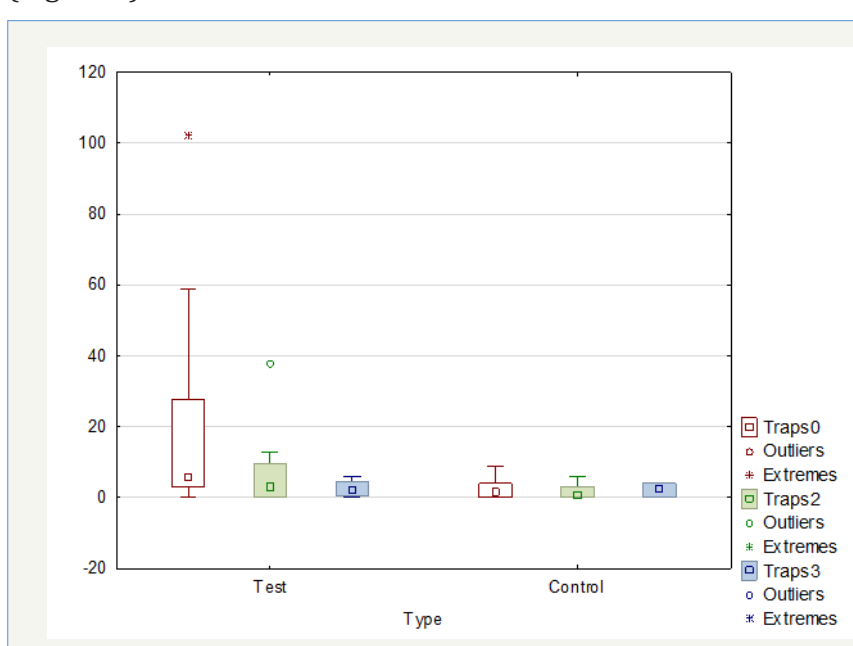

Figure 3: Box plots of number of mosquitoes and median at Test verses Control Sites.

The risk of patients or mosquitoes introducing pathogens into the environment populations is a growing risk in the U.S. Introduced autochthonous cases of malaria have occurred in Georgia. In Bulloch County, travel related cases of Zika, Chikungunya, and dengue, have been documented in recent years with increasing frequency [4-6]. Imported cases of malaria, dengue, Chikungunya, and Zika viruses have occurred in Georgia, and several in Bulloch County 7,2015 , 2016). With the presence of native mosquito species susceptible to malaria and arboviruses in Bulloch County, rapidly reducing mosquito populations surrounding a person's home is an important strategy in reducing the risk of MBD's spreading to other people and possibly becoming endemic. For example, Ae albopictus is an invasive species to southeastern Georgia, and is competent vector of several arboviruses, and may play a role if Zika virus is introduced Kollars et al [3]. Other mosquito species such as, Ochlerotatus japonicas, Culex coronator have also been introduced into Georgia and could expand their range into Bulloch County [8,9]. Very little is known of distribution, mosquito species composition or status of mosquito-borne diseases in Bulloch County.

The City of Statesboro, Bulloch County, has a mosquito control program in the Division of Public Works, but funding is very low for mosquito control (personal communication). A systematic mosquito surveillance effort should be conducted to elucidate the 
species and distribution of mosquitoes and arboviruses in Bulloch County. Citizens living in poor and minority neighborhoods may be at particularly high risk from introduced arboviruses La Deau et al. [7] including neighborhoods in Georgia [10]. When there is the detection of a patient infected with an introduced arbovirus, the City of Statesboro can respond quickly, but must import resources from other counties and the federal government. An example of this was an imported case of Chikungunya into Statesboro; Statesboro relied upon the neighboring Mosquito Control Division of Chatham communication).

Providing inexpensive and ecosafe products may help to protect public health where; mosquito control programs are not available or lack adequate funding and personnel, rapid response with adequate equipment is not available. The ProVector $®$ Tube with Entobac D provides rapid effective control of mosquito populations, while being inexpensive, target specific and environmentally friendly. Additional studies in Bulloch County are being conducted to evaluate new technologies of mosquito control and to identify mosquito species and their distribution in the county [11].

\section{Acknowledgement}

This study was supported in part by the Research Foundation of Georgia Southern University. This manuscript is dedicated to the memory of Peggy Kollars, co-author, mother, wife, scientist, teacher and friend who has gone on to Heaven. The co-authors know that we will see Peggy again because we also have faith in Jesus.

\section{Conflict of Interest}

Prof. Thomas Kollars is the inventor of ProVector ${ }^{\circledR}$ and Entobac $^{\mathrm{TM}}$. County and the CDC to conduct surveillance and control (personal

\section{References}

1. WHO (2017) Vector-borne diseases.

2. Buckner EA, Blackmore MS, Golladay SW, Covich AP (2011) Weather and landscape factors associated with adult mosquito abundance in southwestern Georgia USA. J Vect Ecol 36(2): 269-278.

3. Yalwala S, Kollars JW, Kasembeli G, Chrisostim BW, Senessie C, et al. (2016) Preliminary report on the reduction of adult mosquitoes in housing compounds in western Kenya, using Entobac biopesticide and the ProVector Flower. J Med Ent 83: 1252-1244.

4. Centers for Disease Control and Prevention (1997) Sports-related recurrent brain injuries--United States. MMWR Morb Mortal Wkly Rep 46(10): 224-227.

5. MacArthur JR, Holtz TH, Jenkins J, Newell JP, Koehler JE, et al. (2001) Probable Locally Acquired Mosquito-Transmitted Malaria in Georgia, 1999. Clin Infect Dis 32(8): 124-128.

6. Centers for Disease Control and Prevention (2017) Under case counts of arboviruses for 2013 through 2017.

7. LaDeau SL, Leisnham PT, Biehler D, Bodner D (2013) Higher Mosquito Production in Low-Income Neighborhoods of Baltimore and Washington, DC: Understanding Ecological drivers and mosquito-borne disease risk in temperate cities. Int J Environ Res Public Health 10(4): 1505-1526.

8. Gray EW, Harrison BA, Womack ML, Kerce J, Neely CJ, et al. (2005) Ochlerotatus japonicus japonicus (Theobald) in Georgia and North Carolina. J Am Mosq Cont Assoc 21(2): 144-146.

9. Mullis RA, Russell JD, Lewandowski HB, Thompson PS, Heusel JL, et al. (2008) Culex coronator in Coastal Georgia and South Carolina. J Am Mosq Cont Assoc 24(4): 588-590.

10. Kollars TM (2017) Identifying Health Disparities of Mosquito-Borne Diseases in Low and Medium Income Communities in Savannah, Georgia, USA. Ann Com?munity Med Pract 3(2): 1-3.

11. Kollars TM, PG Kollars, B Hulsey (2016) Reducing the risk to marine ports from invasive mosquito species, Zika, dengue, chikungunya viruses and filariasis. Intl J Med 4(2): 70-73.

\section{Your subsequent submission with Crimson Publishers will attain the below benefits}

Creative Commons Attribution 4.0

International License

For possible submissions Click Here

Submit Article 